\title{
Tidal expired airflow patterns in adults with airway obstruction
}

\author{
E.M. Williams, R.G. Madgwick, M.J. Morris
}

Tidal expired airflow patterns in adults with airway obstruction. E.M. Williams, R.G. Madgwick, M.J. Morris. @ERS Journals Ltd 1998.

ABSTRACT: Earlier studies have shown that time and flow indices derived from tidal expiratory flow patterns can be used to distinguish the severity of airway obstruction. This study was designed to address two aspects of tidal expiratory flow patterns: 1) how do expiratory flow patterns differ between subjects with normal and obstructed airways; and 2) can a sensitive index of airway obstruction be derived from these pattern differences?

Tidal expiratory flow patterns from 66 adult subjects with varying degrees of airway obstructive disease with a forced expiratory volume in one second (FEV1) of 20$121 \%$ predicted were examined. In each subject, the expired flow pattern from each consecutive breath was scaled and then averaged together to create a single expired pattern.

A detailed examination of the scaled flow patterns in 12 subjects (six with normal airways and six with airway obstruction) showed that the shape of the post-peak expiratory flow portion was different in the subjects with airway obstruction. A slope index, $\bar{S}$, was derived from the scaled patterns and found to be sensitive to the severity of airway obstruction, correlating with FEV1 (\% pred) with $\mathrm{r}^{2}=0.74(\mathrm{p}<0.05, \mathrm{n}=57)$. The $\overline{\mathrm{S}}$ index also correlated $\left(\mathrm{r}^{2}=0.36, \mathrm{p}<0.05, \mathrm{n}=47\right)$ with the functional residual capacity (FRC) (\% pred) which was $>\mathbf{1 0 0 \%}$ in subjects with severe airway obstruction and lung overinflation. In subjects with normal airways, three further airflow patterns could be distinguished, which were different from the patterns seen in subjects with the severest airway obstruction.

Scaled flow patterns from tidal expiration collected from uncoached subjects, can be used to derive an index of airway obstruction.

Eur Respir J 1998; 12: 1118-1123.
Lung Function Laboratory, Osler Chest Unit, Churchill Hospital, Oxford, UK.

Correspondence: E.M. Williams

Lung Function Laboratory

Osler Chest Unit

Churchill Hospital

Oxford OX3 7LJ

UK

Fax: 441865225221

Keywords: Airway obstruction

expiration

tidal breathing

Received: October 21997

Accepted after revision May 101998

E.M. Williams was supported by funds from the Oxford Radcliffe NHS Trust Charitable Fund and from Oxfordshire Health Service Research Funds. R.G. Madgwick was supported by Allen and Hanburys.
The observation that the expired airflow pattern changes according to the severity of airway obstruction was reported by MoRris and LANe [1]. The realization that this observation is of practical advantage in offering a simple method of measuring airway obstruction has led to a series of studies both in adults and children [2-5].

The expired airflow pattern seen in people with chronic obstructive airway disease (COAD) differs from the airflow pattern of people with no COAD (fig. 1) by exhibiting a more rapid rise to peak flow, which is followed by a slower rate of decay. The flow patterns also differ at the end of expiration. The pattern from the COAD subjects ends by a sudden deceleration of flow. The point at which this change occurs has been shown in other studies to relate to the degree of lung overinflation [6].

A previous study [1] has described the relationship between airway obstruction and the time to reach peak tidal expiratory flow, $t$ PTEF. In obstructed airways, peak expiratory flow is reached sooner than in those with normal airways. This more rapid rise in expiratory flow is thought to be due to a faster decline in post-inspiratory muscle activity $[7,8]$. As a consequence, a greater part of expiration is passive in subjects with airway obstruction. A good correlation between the ratio, $t \mathrm{PTEF} /$ total expiratory time $(t \mathrm{E})$ and the forced expiratory volume in one second (FEV 1 ) has been found in school-aged children (aged 3-18 yrs), and can be used to distinguish those with asthma and with cystic fibrosis from children with no airway obstruction [9]. In younger children (mean age $<3$ yrs) the same ratio was able to distinguish asthmatics from nonasthmatics [10]. In babies, expired airflow patterns are more variable and the link between $t \mathrm{PTEF}$ and lung mechanics is more complex [4, 5, 11-13]. In adults, further indices of airway obstruction have been derived from the expired patterns $[1,2,6]$. These indices are derived from an analysis of the decaying flow pattern after the peak, and describe the rate of decay in flow and the degree of lung overinflation. These indices correlate well with the severity of airway obstruction, but require some degree of subjective interpretation as to which portion of the decay curve to analyse.

The variability of breathing patterns between breaths and subjects has not been approached systematically in previous studies, so the initial aim of this study was to describe and categorize the variety of airflow patterns seen in normal and obstructed airways. The second aim was to find a simple and robust predictor of airway obstruction from the differently shaped airflow patterns that can be derived easily and objectively. Using tidal airflow patterns to assess the severity of COAD will allow measurements to be made without the subject having to make specific respiratory manoeuvres. 

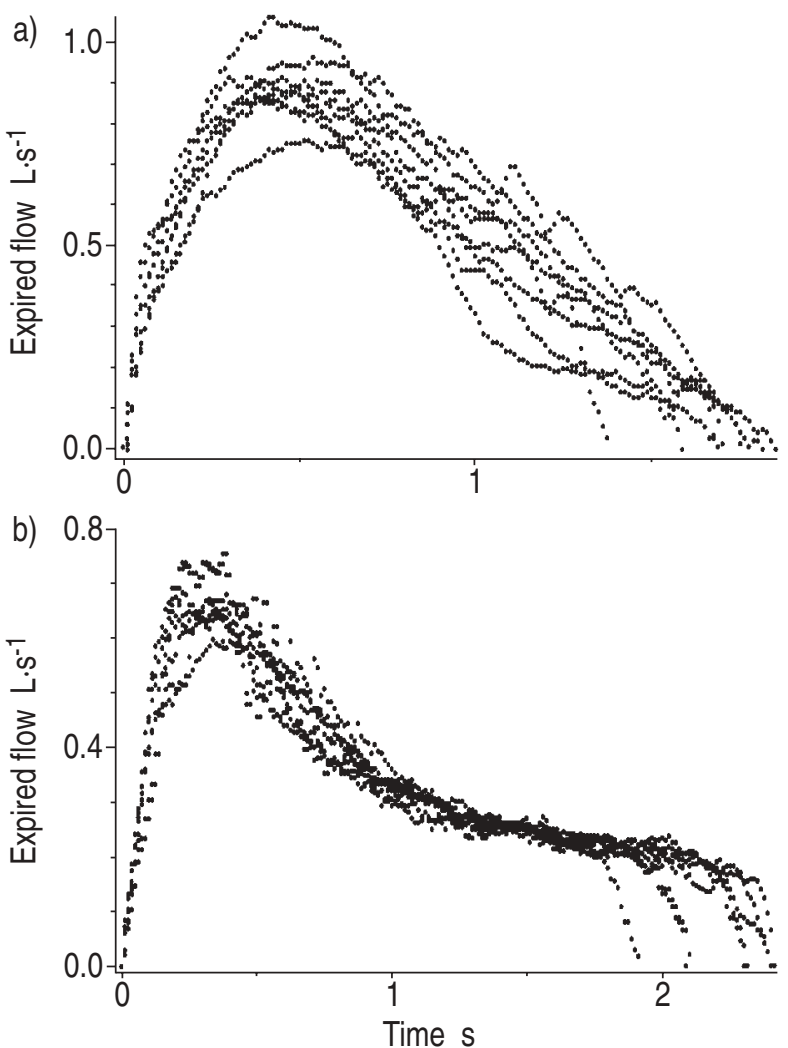

Fig. 1. - The expired flow patterns of 8 consecutive breaths in two subjects, one with a) normal airways (FEV1 111\% pred); and one b) with severe airway obstruction (FEV1 24\% pred). FEV1: forced expiratory volume in one second.

\section{Methods}

Expired airflow patterns were analysed in 66 subjects (24 males, 42 females, aged 18-80 yrs) using data collected for the accompanying study [6].

Tidal flow was recorded from seated uncoached subjects breathing through a mouthpiece connected in series with a calibrated Fleisch pneumotach (P.K. Morgan, Rainham, Kent, UK) with a dead space of 0.12 L [6]. The collection of tidal flow data began within a few seconds of inserting the mouthpiece and placing the noseclip. A mean $( \pm S D)$ of $12 \pm 3(n=66)$ breaths was collected from each subject and data stored on a computer. Before the flow data were collected, each subject underwent spirometry so that their dynamic lung volumes could be measured. Functional residual capacity (FRC) was measured in 46 subjects via whole-body plethysmography [6]. The severity of airway obstruction was classified according to the subject's FEV1 \% predicted (table 1 ).

The stored airflow patterns were analysed by computer, so that for each breath the time expiration started and ended and the expiratory flow reached its peak ( $t \mathrm{PTEF})$ were defined. Finding these "landmark" points allowed further manipulation of the expired flow and time data by computer.

\section{Analysis of expired airflow patterns}

Initially, a subset of 12 subjects was analysed in detail. This group consisted of six subjects with normal airways (FEV1 $103 \pm 7 \%$ pred, mean \pm SD), and six with severe air-
Table 1. - Classification criteria for airway obstruction on severity based on forced expiratory volume in one second (FEV1): severity index I

\begin{tabular}{lc}
\hline Severity index & FEV1 $\%$ pred \\
\hline 1 Normal & $>79$ \\
2 Mild & $61-79$ \\
3 Moderate & $41-60$ \\
4 Severe & $<41$ \\
\hline
\end{tabular}

$\%$ pred: percentage of predicted value.

way obstruction (FEV1 29 $\pm 7 \%$ pred). For each group, subjects were selected on the basis of each having similar airflow patterns and FEV 1 values. The purpose of this selection procedure was to polarize the differences between the patterns from each group. Figure 1 shows eight overlaid expired flow patterns that were collected consecutively from two subjects, one with normal airways (fig. 1a) and the other with obstructed airways (fig. 1b). In each of the 12 subjects, the expired flow patterns from each breath (as shown in fig. 1) were averaged together to form single plots (figs. $2 \mathrm{a}$ and $3 \mathrm{~b}$ ). Figures $2 \mathrm{~b}$ and $3 \mathrm{~b}$ also show the same expired flow patterns after scaling the flow and time data. The flow axis was scaled, so that the peak expiratory flow equalled $100 \%$ and zero flow $0 \%$. To scale the time axis, the start was designated as $0 \%$ and the end of the pe-riod being examined as $100 \%$ (figs. $2 b$ and $3 b$ ). The scaled flow patterns were divided into two portions, the first portion represented the flow pattern before reach-
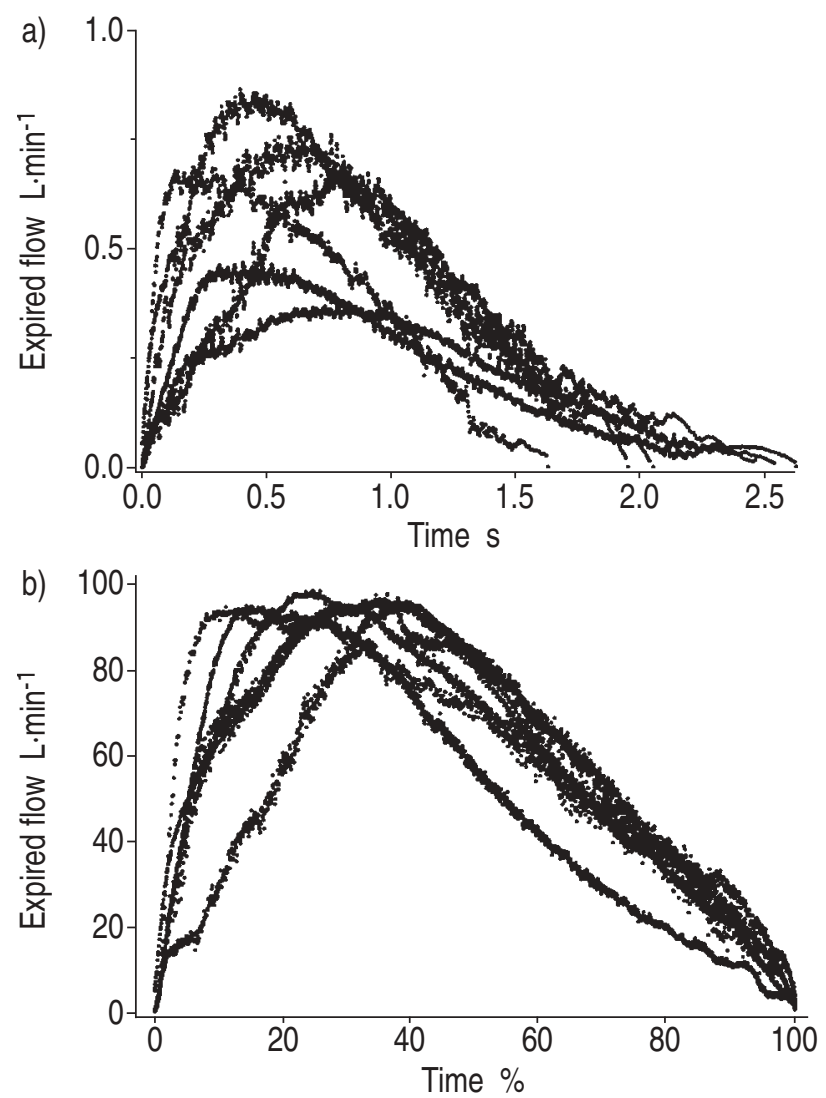

Fig. 2. - Mean expired flow patterns in six subjects with normal airways (mean forced expiratory volume in one second $103 \pm 7 \%$ pred). Each plot shows the mean flow pattern for all of the breaths collected from each subject. a) Variation in flow and duration of expiration between subjects; and b) the same data but with scaled flow and time axes. 

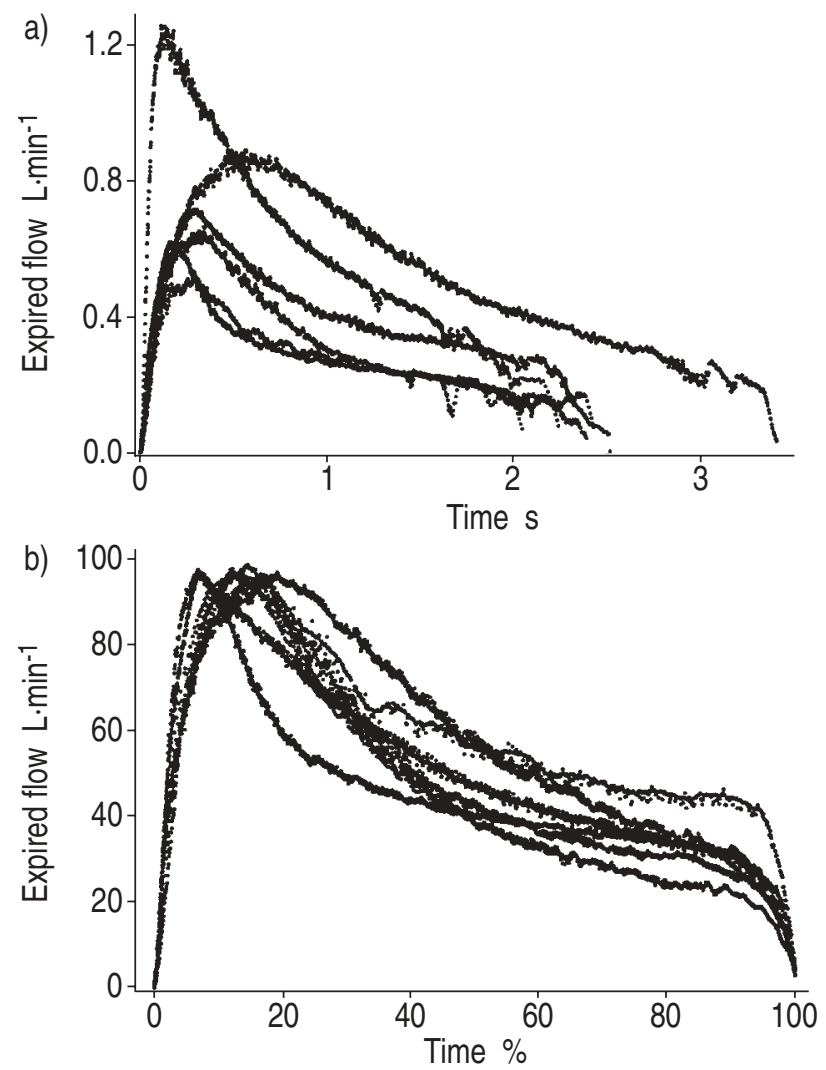

Fig. 3. - Mean expired flow patterns in six subjects with severe airway obstruction (mean forced expiratory volume in one second $29 \pm 7 \%$ pred). a) Variation in flow and duration of expiration between subjects; and b) the same data with scaled flow and time axes.

ing the time of peak tidal expiratory flow, designated as, pre-tPTEF. The second portion was the post-peak expiratory flow pattern, defined as post- $t$ PTEF. In the first portion the flow was re-scaled so that the beginning of expiration was $0 \%$ and the peak expiratory flow rate was $100 \%$; the time axis was rescaled likewise. For the second portion, the flow was rescaled so that the peak expiratory flow rate was $100 \%$ and the end of expiratory flow $0 \%$. On the time axis the $t$ PTEF was defined as $0 \%$ and the time at the end of expiration, $100 \%$ (fig. 4).

\section{Results}

An analysis of the scaled expired flow patterns, from the 12 subjects in figures $2 \mathrm{~b}$ and $3 \mathrm{~b}$, shows that the $t$ PTEF $(\%)$, was significantly shorter $(\mathrm{p}<0.05$, unpaired $\mathrm{t}$-test) in subjects with COAD, $12 \pm 4$ compared with $26 \pm 11 \%$ in those with no airways disease. The correlation between $t$ PTEF $(\%)$ and FEV 1 (\% pred) produced an $\mathrm{r}^{2}=0.40(\mathrm{p}<$ $0.05, \mathrm{n}=12$ ). Thus, the slope of the line describing the rise from the start of expiration to peak expiratory flow $(t \mathrm{PTEF})$ will also be significantly $(\mathrm{p}<0.05)$ steeper in the COAD subjects and was $9.1 \pm 3.6$ compared with $4.5 \pm$ 2.5 in the subjects with no airway disease. When the pre$t$ PTEF portion was analysed and rescaled separately, thus removing the differences between $t$ PTEF (fig. 4a), the slope and intercept on the flow axis of a linear regression line fitted through the whole data (0-100\% time) was found to be independent of the severity of airway obstruc-
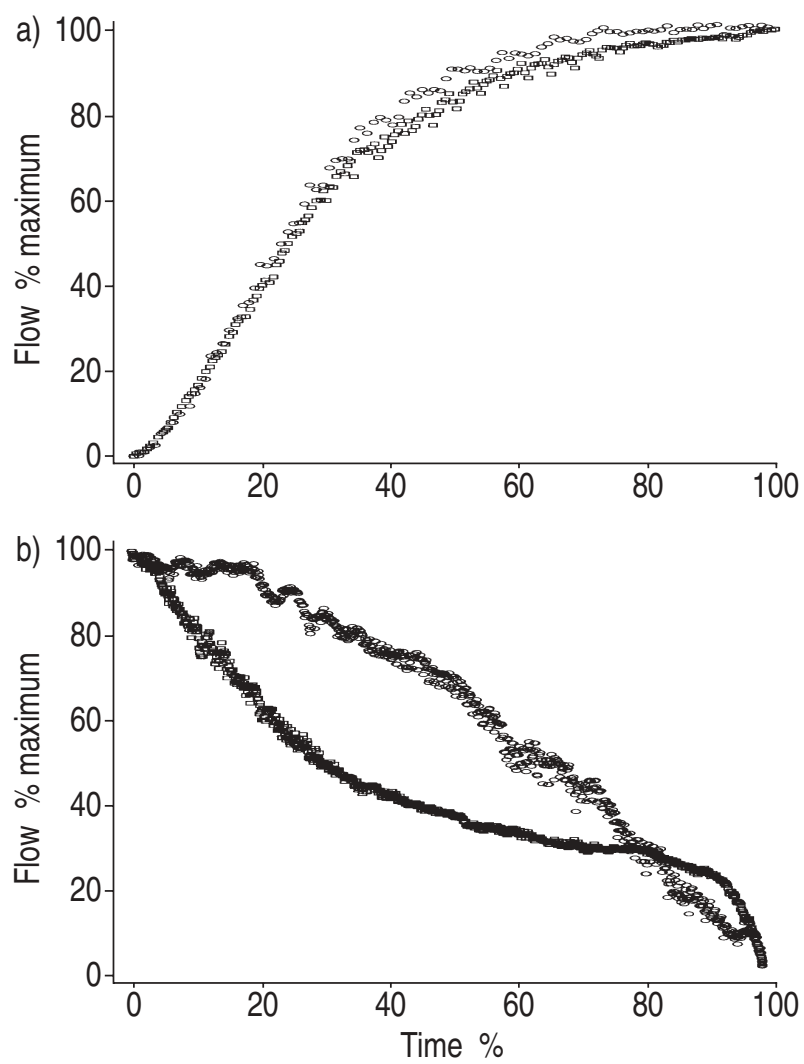

Fig. 4. - Examples of the a) pre-peak expiratory flow portion and b) post-peak expiratory flow portion of the scaled and averaged low pattern from the individual subjects shown in figure 1, one with normal airways (o) and another with severe airway obstruction ( $\square$ ).

tion. No further analysis was performed on this portion of the flow pattern in the other 54 subjects. The same analysis of the second portion of the flow pattern shown in figure $4 \mathrm{~b}$, the post-tPTEF pattern, was different between subjects and was found to be dependent upon the severity of airway obstruction. The fitted regression line through this portion of the scaled flow and time data (fig. 5) describes the mean slope of each pattern, $\overline{\mathrm{S}}$. The differences between $\overline{\mathrm{S}}$ were significant $(\mathrm{p}<0.05)$, with a mean $\mathrm{S}^{-}$of $0.62 \pm 0.11$ in subjects with COAD and $-0.97 \pm 0.04$ in the subjects without COAD. The correlation between $\overline{\mathrm{S}}$ and FEV1 \% pred was $\mathrm{r}^{2}=0.83(\mathrm{p}<0.05, \mathrm{n}=12)$. As in these 12 selected subjects, $\overline{\mathrm{S}}$ provided a good correlation with $\mathrm{FEV} 1$ $\%$ pred; $\overline{\mathrm{S}}$ was then derived from the patterns of a further 54 subjects (table 2 ).

\section{Post-peak expiratory flow patterns}

Classification of the 66 subjects into four groups of differing airway obstruction (table 1 ) showed that $\bar{S}$ became smaller with increasing airway obstruction (i.e. decreasing FEV1 \% predicted) (table 2). The inclusion of subjects with a wider range of airway obstruction worsens the correlation between $\overline{\mathrm{S}}$ and FEV $1 \%$ pred, with $\mathrm{r}^{2}=0.42(\mathrm{p}<$ $0.05, n=66$ ). A closer examination of the data revealed that this was due to a small group of subjects with normal airways having an $\mathrm{S}$ value similar to subjects with severe airway obstruction. A more detailed look at the expiratory patterns of subjects with normal airways (FEV $1>79 \%$ pred, table 2) showed that the scaled expired flow patterns 


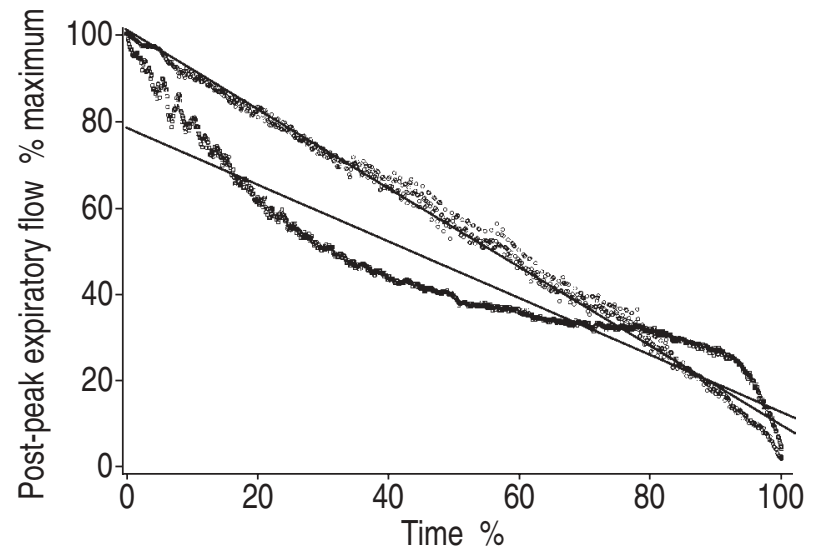

Fig. 5. - The post-peak expired flow portion from the individual subjects shown in figure 1 , one with normal airways ( 0 ) and one with severe airway obstruction $(\square)$. A linear regression line is fitted through each data set, the slope $\bar{S}$ and intercept on the flow axis for the subject with normal airways is -0.9 and $101 \%$ and for the subject with severe airway obstruction it is -0.7 and $179 \%$.

could be split into three distinct groups. The commonest group (62.5\%) exhibited a sinusoidal flow pattern with a linear decline in their expiratory flow with time, and these were classified as type I breathers (fig. 6a). A second, less common, group $(12.5 \%)$ were classified as type II breathers, with a characteristic of these patterns being a rapid decline in post-peak flow with an expiratory pause before the beginning of inspiration (fig. 6b). These expiratory pat-terns result from relaxed breathing, where no expiratory muscle braking occurs. In a third group (25\%), classified as type III breathers, the expiratory flow remained high throughout most of the breath before suddenly switching to inspiration (fig. 6c). The shape of the expiratory patterns from type II breathers was concave in relation to the type I breathers, while the shape of the expiratory patterns in type III breathers was the reverse and exhibited a convex relationship. The type III patterns, although having a similar $\mathrm{S}$, are distinctly different from the patterns exhibited by any other subjects including those with severe COAD, and so they were analysed separately (see Discussion).

Figure 7 shows the relationship between FEV1, and $\mathrm{S}$ for subjects with different airflow patterns and varying degrees of airway obstruction. By excluding the data from the type III breathers, a fitted quadratic regression line

Table 2. - Expired flow pattern characteristics in 66 subjects

\begin{tabular}{lcccc}
\hline & \multicolumn{4}{c}{ Airways obstruction severity index } \\
\cline { 3 - 5 } Parameters & $\begin{array}{c}\text { Normal* } \\
(\mathrm{n}=32)\end{array}$ & $\begin{array}{c}\text { Mild } \\
(\mathrm{n}=7)\end{array}$ & $\begin{array}{c}\text { Moderate } \\
(\mathrm{n}=10)\end{array}$ & $\begin{array}{c}\text { Severe } \\
(\mathrm{n}=17)\end{array}$ \\
\hline FEV1\% pred & $101 \pm 12$ & $68 \pm 6$ & $48 \pm 6$ & $31 \pm 6$ \\
FEV1/FVC \% & $81 \pm 9$ & $68 \pm 8$ & $48 \pm 13$ & $36 \pm 8$ \\
$t \mathrm{PTEF} / t \mathrm{tE} \%$ & $34 \pm 10$ & $33 \pm 8$ & $29 \pm 12$ & $27 \pm 11$ \\
$\overline{\mathrm{S}}$ & $-0.90 \pm 0.13$ & $-0.81 \pm 0.12$ & $-0.78 \pm 0.07$ & $-0.67 \pm 0.11$
\end{tabular}

Values shown as mean \pm SD. See text for statistical comparisons. *: includes type I, II and III breathers. describes the slope of a fitted regression line, $Y=a+b x$. NB. The last two variables were calculated from the scaled data. FEV1: forced expiratory volume in one second; FVC: forced vital capacity; $t$ PTEF: time to reach peak tidal expiratory flow; $t \mathrm{E}$ : total expiratory time; $\overline{\mathrm{S}}$ : slope index. a)
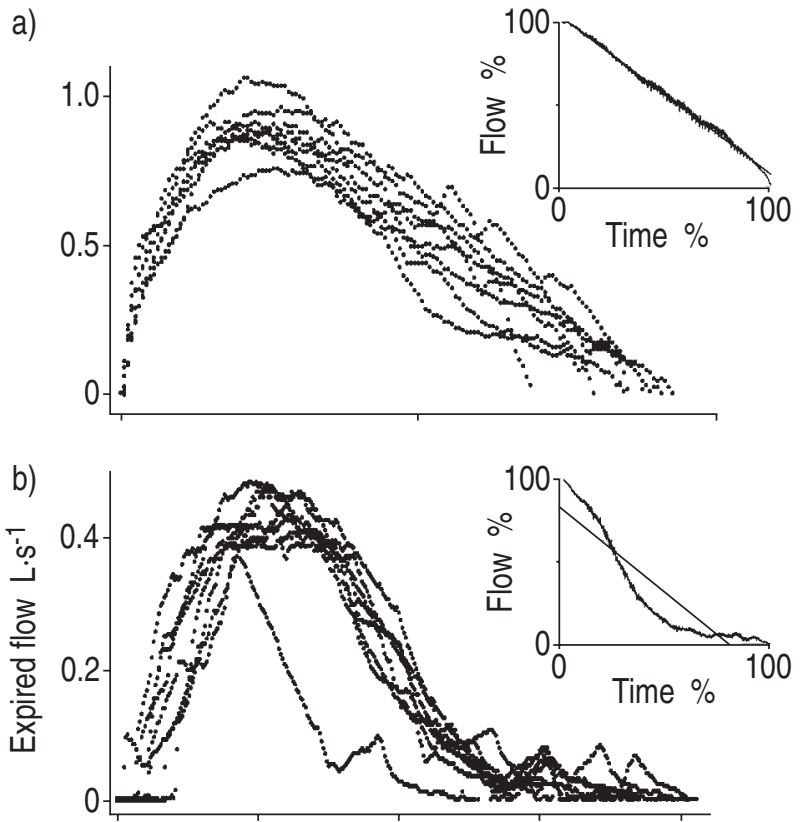

c)

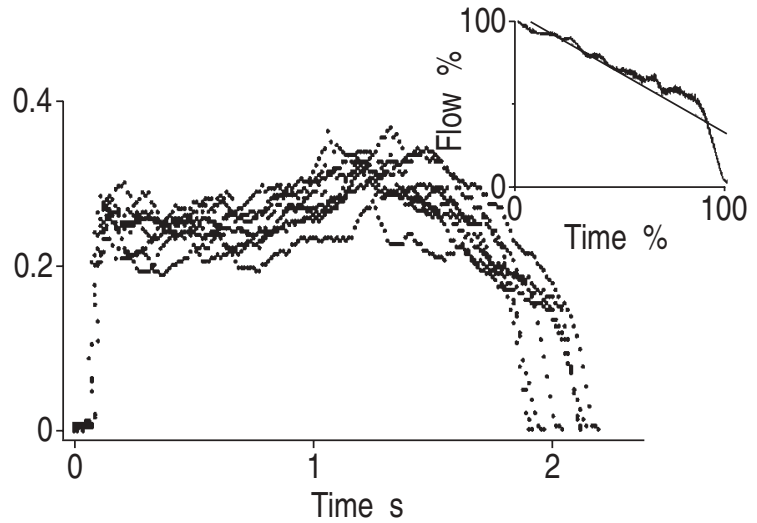

Fig. 6. - Different expired flow patterns in subjects with normal airways. Each example shows eight consecutive breaths from individual subjects. a) Type I pattern, which is sinusoidal in shape (mean forced expiratory volume in one second (FEV1) $111 \%$ pred, slope $\overline{\mathrm{S}}-0.96$ ); b) Type II pattern is characterized by an end-expiratory pause in flow (FEV1 $102 \%$ pred, $\overline{\mathrm{S}}-1.04$ ); and c) Type III pattern is more square in shape, with longer periods of constant flow (FEV1 100\% pred, $\widehat{S}-0.74$ ). Each inset shows the scaled and averaged post-peak expiratory flow pattern for each subject. The abscissa is scaled $0-100 \%$ time, while the ordinate is scaled $0-100 \%$ flow. The line describing $\overline{\mathrm{S}}$ is also shown.

gives an $\mathrm{r}^{2}=0.74(\mathrm{p}<0.05, \mathrm{n}=57)$. If the original 12 subjects are also excluded, $\mathrm{r}^{2}=0.67(\mathrm{p}<0.05, \mathrm{n}=45)$.

In all subjects $(n=66)$, the total expiratory time was independent of the degree of airway obstruction, with the overall mean time being $2.4 \pm 0.3 \mathrm{~s}$. The peak flow rate was also independent of airway obstruction, with an overall mean rate of $0.55 \pm 0.06 \mathrm{~L} \cdot \mathrm{s}^{-1}$.

\section{Lung overinflation}

The degree of lung overinflation was assessed from the post- $t$ PTEF flow pattern by calculating the intercept on the scaled time axis of the fitted regression line, S. This overinflation index is similar to the extrapolated volume index (EV) described by MoRris et al. [6]. The S intercept on the scaled time axis was compared to the predicted FRC (\%), which was calculated as the ratio between the meas- 


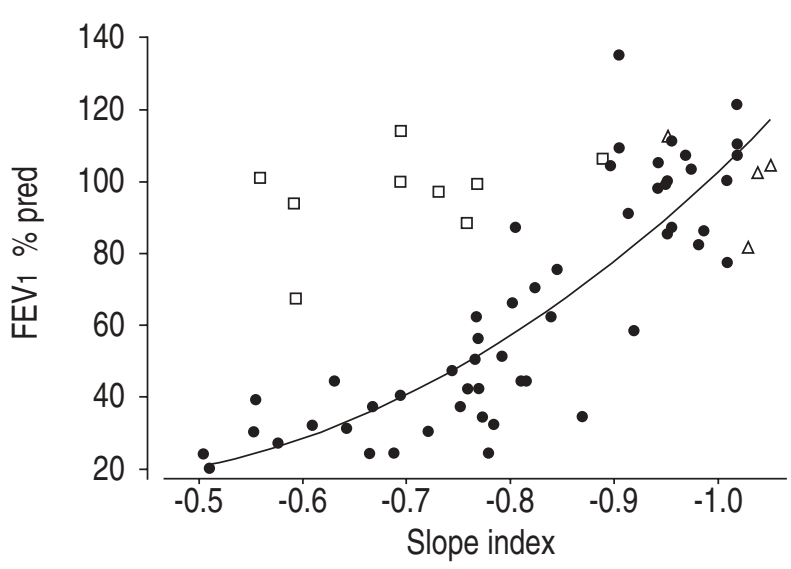

Fig. 7. - The relationship between forced expiratory volume in one second (FEV1) (\% pred) and slope index $(\overline{\mathrm{S}})$ from the post-peak expiratory flow pattern. A quadratic regression line was fitted through the individual data $(\bullet)$ (correlation coefficient $r^{2}=0.74, n=57$ ). Type II $(\Delta)$ and type III ( $\square$ ) breathers are shown separately.

ured and predicted FRC (L). In the 47 subjects who had their FRC measured, the correlation between the S intercept and the predicted FRC was good, with $\breve{S}^{2}=0.37$. The subjects in this group with no COAD had a mean FRC \% pred of $107 \pm 23(n=14)$, which compared closely with an S intercept value of $108 \pm 9 \%$. In the subjects with severe COAD, the FRC \% pred was $176 \pm 46(n=17)$, while the S intercept was $143 \pm 19 \%$.

\section{Discussion}

The expired airflow pattern in both normal and obstructed airways varies with each breath (fig. 1). Figure 1a shows a typical normal airflow pattern from a single subject, in which the peak flow rate varies $0.7-1 \mathrm{~L} \cdot \mathrm{s}^{-1}$ and the duration of expiration varies 1.4-1.9 s. In these examples, the post-peak expiratory flow rate in the normal subject varies more from breath to breath than in the subject with obstructed airways, particularly at $1.5 \mathrm{~s}$. When this relationship was examined across the whole range of subjects, the variability of this portion of the pattern did not provide a good indicator of airway obstruction. There is also variation between subjects who have the same degree of airway patency (figs. $2 \mathrm{a}$ and $3 \mathrm{a}$ ). This interbreath and intersubject variability makes the comparison of primary indicators such as peak flow rate and breath length of limited use in defining the degree of airway obstruction. In this study, the purpose of scaling the flow and time axes was to emphasize common features between expiratory flow patterns that would allow the flow patterns to be classified according to their shape (figs. $2 b$ and $3 b$ ). Once scaled, flow patterns from different subjects could also then be superimposed over any other flow pattern for comparison.

\section{The rise to peak expiratory flow}

These data support an earlier study, which demonstrated that the time to reach peak expiratory flow is shorter in subjects with severe COAD than in those with normal airways [1]. In the present study, the peak expiratory flow rate reached was independent of airway obstruction, therefore a shorter $t$ PTEF in subjects with severe COAD can only be achieved by having a more rapid rise to the ma- ximum flow rate. Because of this rapid rise to peak flow, the variability of $t$ PTEF is smaller in subjects with airway obstruction. In the scaled patterns of the six subjects with severe COAD, $t$ PTEF ranged $7-15 \%$. In the subjects with normal airways, $t$ PTEF is longer and, because the rise to maximum flow is slower, the index range is less well defined. In the six subjects with no COAD, the range in $t \mathrm{PTEF}$ was $11-38 \%$, with half of the $t$ PTEF values overlapping the values found in subjects with severe COAD. Thus, the airflow patterns in the severe COAD subjects are uniform, unlike the patterns in the nonCOAD group which are more variable (figs. $2 b$ and $3 b$ ). It is because of the overlap between the two groups that $t$ PTEF can only be used to distinguish the distribution of airway obstruction within a population, and cannot be used to define airway obstruction in individual cases.

The effect of rescaling the pre- $t$ PTEF portion of the flow pattern was to reduce the variability between airflow patterns. This scaling process rendered this portion of the flow pattern useless for estimating airway obstruction. However, the opposite effect was observed with the post$t$ PTEF data. Scaling both axes enhanced the distinction between the different airflow patterns.

\section{Post-peak expiratory flow}

This portion of the expiratory flow pattern exhibits the greatest variation (figs. 2, 3 and 6), and other studies have used indices derived from this portion to try and quantify the different patterns $[2,6]$. The post-tPTEF flow patterns in the majority of subjects with normal airways (type I breathers) exhibit linear decay rates, so a linear regression model can be easily applied to the data. Thus, the slope of this regression line, $S$, which spans the whole post- $t$ PTEF pattern, provides a simple index of the change in flow rate. However, with increasing severity of airway obstruction, the expired pattern becomes more concave, so a linear function applied to the whole post- $t$ PTEF pattern does not fit so well. The fit of a linear function is of little consequence. What is important is the shallowness of the slope, which increases as the pattern's concavity increases. The shallowness of the slope of the regression is determined by the two factors which distinguish severe from normal airflow patterns, that is the rapid nonlinear decay in flow immediately following $t$ PTEF and the deceleration in flow immediately preceding inspiration. As the airways become more obstructed, the magnitude of these rapid flow changes increase, so the decay in flow seen in the midportion of the expiratory flow pattern becomes shallower and lon-ger in duration. S, thus described, is a good indicator of these airway obstruction-induced changes in pattern be-cause it represents the mean slope. The mean slope results from the sum of the effects of airway obstruction on the mechanical properties of the respiratory system. The ratio $t \mathrm{PTEF} / t \mathrm{E}$ may be a weaker indicator of airway obstruction, because it relies on a single part of the expiratory flow pattern $[1,4,5]$. Thus, when this index is applied to the wide spectrum of patterns found between normal, mild, moderate and severe airway obstruction, the considerable variation and overlap between individuals reduces its predictive power (table 2). Applying a linear model to the whole data set provides a wholly objective measure of airway obstruction. Applying models to a smaller portion of the curve, such as an exponential function, may also provide an indicator of airway obstruction, 
but requires subjective interpretation of the pattern as to where to start and end the fitting process.

$\mathrm{S}$ is not a perfect indicator of airway obstruction, as fig. 7 shows. Type III breathers exhibit similar $\mathrm{S}$ values to the subjects with severe COAD $(-0.71 \pm 0.1, n=8$ and $-0.67 \pm$ $0.11, \mathrm{n}=17$, respectively). However, the type III breathers exhibit a convex pattern in relation to the normal type I breathers (fig. 6). This convex pattern has a low S, as in the severe pattern, but unlike the typical severe COAD pattern, which is concave, the $\mathrm{S}$ intercept on the flow axis is $>100 \%$. Thus, if an additional step is used to distinguish subjects with severe COAD from normal type III breathers, then a severity index of airway obstruction can be constructed (table 3 ).

The indices derived from the FEV1 (tab̄le 1) and S (table 3) can be compared by subtracting one index from the other. If the severity index derived from $\mathrm{S}$ is similar to the FEV1 index, then the difference between them should be zero. In 53 subjects, this is true, with a further 11 subjects misclassified by a single category. In most cases, misclassification by one group is due to the subject's FEV1 and $S$ values falling either side of a category boundary. Two subjects were misclassified by two categories; one severe subject was misclassified as mild and one subject with moderate airway obstruction was misclassified as normal. A close match of 64 subjects out of 66 indicates an agreement between the two severity indices of $97 \%$. The two misclassified subjects had expired airflow patterns that were similar to patterns of these subjects in normal type I breathers. Why these expired airflow patterns of the subjects were different requires further investigation into the aetiology of their airway obstruction.

When deriving $S$, the intercept of this line on the scaled time axis provides a sensitive indicator of dynamic lung overinflation, a common phenomenon seen in subjects with severe airway obstruction [15]. In subjects with no lung overinflation, a linear function provides the best fit to the airflow pattern. This line crosses the flow and time axes close to $100 \%$ (fig. 7a). However, subjects with overinflated lungs tend to have the lowest $\mathrm{S}$ index, so that a fitted linear regression line intercepts the flow axis at approximately $80 \%$ and the time axis at $>100 \%$. The flattening of the post- $t$ PTEF airflow pattern increases with lung overinflation. With severe COAD, the post- $t$ PTEF flow decreases in an exponential manner and overinflation results when there is insufficient time to allow flow to decay to zero. Thus, in overinflation, there must be a rapid deceleration in flow immediately prior to the beginning of inspiration, leading to the flattening of the flow pattern (fig. 5).

In conclusion, widely varying expired airflow patterns, once scaled, can be categorized according to their shape. The shape of the post-peak expiratory flow portion of these patterns is more sensitive to the severity of airway obstruction than the pre-peak expiratory flow portion. A slope index can be derived in a number of easy steps from

Table 3. - Classification criteria for airway obstruction severity based on slope index $(\overline{\mathrm{S}})$ : severity index II

\begin{tabular}{lr}
\hline Severity index & $\overline{\mathrm{S}}$ (negative) \\
\hline 1 Normal & $>0.89$ \\
2 Mild & $0.8-0.89$ \\
3 Moderate & $0.75-0.79$ \\
4 Severe & $<0.75$ \\
\hline
\end{tabular}

this portion of the pattern, which provides a simple and robust indicator of airway obstruction. In deriving this index, the computer first extracts the post-peak expiratory flow portion of each breath collected. The point of maximum expiratory flow is defined and the post-peak portion extracted. The extracted flow and time data are scaled and the data from each breath averaged together to create a unique flow pattern for each subject. The computer then fits a linear regression line through the entire data and the slope of the regression line is calculated. The objective fitting of a regression line by computer requires no prejudgement of the flow patterns as to what category of airway obstruction exists. A further advantage is that the flow data needed to calculate this slope index can be readily collected from uncoached subjects, who need do nothing except breathe quietly through a mouthpiece.

\section{References}

1. Morris MJ, Lane DJ. Tidal expiratory flow patterns in airflow obstruction. Thorax 1981; 36: 135-142.

2. Morris MJ, Madgwick RG, Lane DJ. Analysis of tidal expiratory flow pattern in the assessment of histamineinduced bronchorestriction. Thorax 1995; 50: 346-352.

3. van der Ent CK, Brackel HJL, van der Laag J, Bogaard JM. Tidal breathing analysis as a measure of airway obstruction in children three yrs of age and older. Am J Respir Crit Care Med 1996; 153: 1253-1258.

4. Seddon PC, Davis GM, Coates AL. Do tidal expiratory flow patterns reflect lung mechanics in infants? Am J Res-pir Crit Care Med 1996; 153: 1248-1252.

5. Lødrup Carlsen KC. Tidal breathing analysis in infants and preschool children: tidal flow volume loops. Eur Respir Mon 1997; 5: 27-57.

6. Morris MJ, Madgwick RG, Collyer I, Denby F, Lane DJ. Analysis of expiratory tidal flow patterns as a diagnostic tool in airflow obstruction. Eur Respir J 1998; 12: 1113 1117.

7. Morris MJ, Madgwick RG, Frew AJ, Lane DJ. Breathing muscle activity during expiration in patients with chronic airflow obstruction. Eur Respir J 1990; 3: 901-909.

8. Citterio G, Agostoni E, A DelSanto, Marazzini L. Decay of inspiratory muscle activity in chronic airways obstruction. J Appl Physiol: Respir Environ Exercise Physiol 1981; 51: 1388-1397.

9. Cutrera R, Filtchev SI, Merolla R, Willim G, Haluszka J, Ronchetti R. Analysis of expiratory pattern for monitoring bronchial obstruction in school-age children. Pediatr Pulmonol 1991; 10: 6-10.

10. Carlsen K-H, Lødrup Carlsen KC. Tidal breathing analysis and response to salbutamol in awake young children with and without asthma. Eur Respir J 1994; 7: 2154-2159.

11. Aston H, Clarke J, Silverman M. Are tidal breathing indices useful in infant bronchial challenge tests. Pediatr Pulmonol 1994; 17: 225-230.

12. Lødrup Carlsen KC, Magnus P, Carlsen K-H. Lung function by tidal breathing in awake healthy newborn infants. Eur Respir J 1994; 7: 1660-1668.

13. Stocks J, Dezateux CA, Jackson EA, Hoo A-F, Costeloe $\mathrm{KL}$, Wade AM. Analysis of tidal breathing parameters in infancy: how variable is $t \mathrm{PTEF}: t \mathrm{E}$ ? Am J Crit Care Med 1994; 150: 1347-1354.

14. Martinez FD, Morgan WJ, Wright AL, Holberg CJ, Taussig LM. Diminished lung function as a predisposing factor for wheezing respiratory illness in infants. $N$ Engl $J$ Med 1988; 319: 1112-1117.

15. Rossi A, Ganassini A, Polese G, Grassi V. Pulmonary hyperinflation and ventilator-dependent patients. Eur Respir J 1997; 10: 1663-1674. 